\title{
Déficit de memoria operativa en los trastornos del neurodesarrollo*
}

\section{Working memory deficits in neurodevelopmental disorders}

\author{
Xochitl Delgado Mckay \\ Maryoris Zapata Zabala \\ Universidad San Buenaventura, Medellín, Colombia
}

Artículo de Revisión

Copyright 2018 by Psicogente

Correspondencia de autores:

xochitld27@gmail.com

maryoris.zapata@usbmed.edu.co

\section{Palabras Claves:}

Memoria operativa, Trastornos del neurodesarrollo, Memoria, Memoria de corto plazo,

Neurodesarrollo, Trastornos.

\section{Resumen}

El presente artículo es una revisión sistemática que busca presentar el estado actual de este proceso Cognitivo en los diferentes trastornos del neurodesarrollo, se retomó el constructo de memoria Operativa de Baddeley y Hitch (1974), teniendo en cuenta su desarrollo, los sistemas que lo componen y las diferentes funciones que realiza este proceso cognitivo en el ser humano.

Seguido de esto se continúa con la presentación de los diferentes trastornos del neurodesarrollo Según el Manual Diagnóstico y Estadístico de los Trastornos Mentales y la prevalencia de estos en Colombia. Por último, se analizará la memoria operativa en cada trastorno del neurodesarrollo, Cuáles son los componentes más afectados en cada uno de ellos y las posibles causas que se ven Asociadas a la alteración.

\begin{abstract}
This review paper aims to show the current status of the cognitive domain in different process of the human being.

Furthermore, we will submit different neurodevelopmental disorders based on the Diagnostic and possible causes for such alterations will be analyzed.

Key words:

Working memory, Neurodevelopmental disorders, Memory, Short-term memory,

Neurodevelopmental, Disorder
\end{abstract} neurodevelopmental disorders. As the working memory temporarily stores information to achieve shortterm and immediate objectives, we are going to use the working memory construct by Baddeley and Hitch taking into account, its development, different systems and functions related to the cognitive Statistical Manual of Mental Disorders data and its prevalence in Colombia. Lastly, the working memory in each neurodevelopmental disorders, explaining which components are affected the most and the

Como citar este artículo (APA):

Delgado, X. \& Zapata, M. (2018). Déficit de memoria operativa en los trastornos del neurodesarrollo. Psicogente, 21(39), $216-227$.

http://doi.org/10.17081/psico.21.39.2832 


\section{INTRODUCCIÓN}

La memoria operativa es un tipo de memoria que almacena la información temporalmente para trabajar con ella y así alcanzar objetivos inmediatos y a corto plazo; además sirve para resolver problemas usando la información de manera activa. Esta memoria es limitada y susceptible a interferencias, sin embargo tiene una enorme flexibilidad que permite al sujeto estar siempre Abierto al ingreso de nueva información. Así mismo este proceso cognitivo es importante para la comprensión del lenguaje, para procesar rápidamente la información y para mantener los objetivos en la resolución de problemas (Baddeley, 2012; Etchepareborda \& Abad-Mas, 2005; Flores \& Ostrosky-Shejet, 2012).

Uno de los modelos más utilizados para explicar el constructo de memoria operativa es el propuesto por Baddeley y Hitch (1974), quienes presentan un modelo que consta de tres componentes: el Ejecutivo Central (EC) con dos sistemas subsidiarios, el bucle fonológico (BF) y la agenda visoespacial (AV). Sin embargo, los autores consideraban que faltaba un componente en este modelo, un factor que tuviera la capacidad para integrar este sistema multicomponencial con la memoria de largo plazo; es aquí cuando entra el denominado búfer episódico (Gathercole \& Baddeley, 1993). Se puede definir al ejecutivo central como un sistema atencional de capacidad limitada que selecciona y opera con varios procesos de control, manipulando material en otros subsistemas y controlando diferentes actividades (Baddeley \& Eysenck, 2010; Flores \& Ostrosky-Shejet, 2012). Mientras que el bucle fonológico (BF) es el encargado de procesar la información verbal que para poder mantenerla activa necesita de la repetición o del ensayo ya sea de forma abierta o de forma encubierta. El BF contiene dos subsistemas, un almacén fonológico y uno de recapitulación articulatorio; el primero a través de la repetición subvocal mantiene la información activa mientras que el segundo toma el material presentado visualmente, como palabras o imágenes nombrables y los registra en el almacén fonológico por subvocalización. De la aplicación de este modelo en la memoria de los niños, se ha visto que el bucle fonológico está presente y funcionando en ellos desde los años preescolares y que la expansión de la capacidad de la memoria en el desarrollo del niño se da en gran parte al incremento con la edad de la tasa de ensayo subvocal (Gathercole \& Baddeley, 1993).

Con respecto a la agenda visoespacial, es el componente de la memoria operativa encargado de mantener temporalmente la información visual y espacial (Flores \& Ostrosky-Shejet, 2012). Es utilizado para planificar movimientos y está implicado en la aptitud espacial al igual que en tareas que implican la memoria espacial, como el ajedrez (Etchepareborda \& Abad-Mas, 2005).

Se ha propuesto que la agenda visoespacial está constituida por componentes visuales encargados del procesamiento de patrones y de detectar "el qué", componentes espaciales cuya función es la localización en el espacio y transmite información sobre "el dónde" y probablemente también componentes cinéticos (Arteaga \& Pimienta, 2006).

Por último, está el cuarto componente de la MO, el búfer episódico encargado de crear una relación entre los tres sistemas y la memoria de largo plazo. Este sistema tamiza toda la información basada en su categoría ya sea verbal, visual o perceptual; y aunque es un sistema temporal en su naturaleza puede acceder a información de la memoria de largo plazo ya sea para aprender o para recuperar (Saeed, 2011). 


\section{METODOLOGÍA}

El presente estudio está basado en una revisión sistemática que explora el estado de la memoria operativa en los diferentes trastornos del neurodesarrollo. Una búsqueda de literatura científica fue realizada, tanto en inglés como en español, en diferentes bases de datos bibliográficos internacionales como PubMed, Google Scholar, Science Direct, Springer Journals y Dialnet utilizando palabras como "memoria operativa", "funciones ejecutivas", "autismo", "discapacidad cognitiva", "trastornos del aprendizaje", "trastorno por déficit de atención con hiperactividad", "trastornos del lenguaje". Se tomaron en cuenta estos criterios de inclusión para los estudios en la revisión: 1) Estudios en población infantil o adolescente donde se haya estudiado la memoria operativa en los trastornos del neurodesarrollo, 2) Estudios en español o inglés. Mientras que en los criterios de exclusión estaban: 1) Estudios realizados en población adulta, 2) Artículos en donde el objeto de estudio eran otros procesos cognitivos, 3) Estudios en idiomas que no fueran inglés o español, 4) Artículos de otros trastornos de niños. Se identificaron 79 artículos potencialmente relevantes para esta revisión de los cuales se excluyeron 25 por no cumplir los criterios de inclusión. Al final se utilizaron 51 artículos científicos de 1998 al 2015. Sin embargo, para complementar la información revisada en los artículos se utilizaron referencias más de libros sobre memoria operativa. En total 51 artículos y libros utilizados como información científica para la construcción de este artículo.

\subsection{Trastornos del Neurodesarrollo (TND)}

La memoria operativa se encuentra afectada en los trastornos del neurodesarrollo, esto sucede porque este proceso necesita la adecuada integración de distintos sistemas corticales y subcorticales que están extensamente distribuidos por el cerebro para trabajar efectivamente (Saeed, 2011). De acuerdo al DSM-V se pueden definir los TND como aquellos trastornos que tienen su origen en la niñez, usualmente antes del inicio de la etapa escolar, y que se caracterizan por déficits en el desarrollo que producen limitaciones en áreas específicas o globales produciendo dificultades en distintas áreas como la personal, social, académica o en el funcionamiento ocupacional de la persona (American Psychiatric Association, 2013). Dado los cambios en este manual, se ha agrupado en los Trastorno del Neurodesarrollo a las discapacidades intelectuales, Trastornos de la comunicación, Trastorno del espectro autista, Trastorno por déficit de atención con hiperactividad, Trastornos específicos del Aprendizaje, Trastornos motores y otros trastornos del neurodesarrollo.

Los trastornos del neurodesarrollo presentan una alta prevalencia en Colombia. El trastorno que más se presenta en la población infantil es la discapacidad cognitiva. Según un reporte presentado en el 2010 se encuentra que por cada mil niños registrados de cero a cuatro años, 5,5\% de ellos son niños con discapacidad mientras que en los menores de 5 a 9 años la tasa es del 10,0 \%. En los niños de 10 a 14 años del 12,1 \% en tanto que los jóvenes de 15 a 19 años se presenta una tasa de 10,7 \% (Julio \& Gómez, 2010). Después el que mayor prevalencia presenta es el TDAH con una incidencia de $16,1 \%$ en la población colombiana (Vélez-Álvarez \& Vidarte, 2012). Seguido del TDAH se encuentra el trastorno del espectro autista que, según la Liga Colombiana del Autismo, se presenta en 1 de cada 110 niños en este país.

Sobre los trastornos del aprendizaje y el trastorno del lenguaje no hay estudios sobre su prevalencia en Colombia, sin embargo en Estados Unidos se reporta que el 1,7 \% de la población padece de 
trastornos del aprendizaje. La mayoría de casos son de hombres y es más probable que desarrollen trastornos del aprendizaje aquellas personas que viven en la pobreza. En niños de edad escolar, se reporta una incidencia de 2,2 \% (National Center for Learning Disabilities, 2014).

\subsection{Trastornos del Espectro Autista}

Se definen como graves alteraciones del neurodesarrollo debido a un desarrollo anormal del cerebro, ya sea en etapas prenatales y/o en los primeros años de vida que producen déficits cognitivos que permanecen durante la vida (Pineda, 2014).

En los trastornos del neurodesarrollo se ha encontrado que la memoria operativa en los niños con trastornos del espectro autista, variables como el coeficiente intelectual $(\mathrm{Cl})$ y edad juegan un papel importante para el adecuado desempeño de este proceso cognitivo. En un estudio comparativo realizado por Saeed (2011) se encontró que los niños con trastorno del espectro autista de bajo funcionamiento se desempeñaron peor que los niños con desarrollo normal, sin embargo cuando se controlaban las variables de $\mathrm{Cl}$ y edad no se encontraban diferencias significativas entre los niños de alto funcionamiento con trastorno del espectro autista y el grupo control; lo que sugiere que el $\mathrm{Cl}$ tiene un rol dominante en el funcionamiento de la MO. Sin embargo, en una revisión realizada por Kercood, Grskovic, Banda y Begeske (2014), exponen que los niños con trastorno del espectro autista de alto funcionamiento presentan más cantidad de errores, utilizan menores estrategias y presentan un pobre desempeño en las tareas de memoria operativa en comparación con los niños con un desarrollo normal (Corbett, Constantine, Hendren, Rocke, \& Ozonoff, 2010). Aunque generalmente se ha visto que los niños con trastorno del espectro autista presentan dificultades en el lenguaje, en la revisión realizada por Kercood et al. (2014) se encontró que el componente verbal de la memoria operativa no se ve afectado y que los niños con trastorno del espectro autista presentan un subsistema de recapitulación articulario similar al de los niños con un desarrollo normal. Sin embargo, Goldstein, Carpenter y Minshew (2005) encontraron un peor desempeño en el componente espacial de la MO de los pacientes diagnosticados con trastornos del espectro autista y aquellos sujetos con desarrollo normal, estos déficits se vieron reflejados en tareas que requerían una mayor carga cognitiva y flexibilidad mental.

\subsection{Trastorno por Déficit de Atención con Hiperactividad (TDAH)}

Es una condición clínicamente diversa cuyos rasgos principales son la desatención, la hiperactividad y la impulsividad. Era considerado como un trastorno propio de la infancia que disminuía o desaparecía en la adolescencia; sin embargo, el seguimiento a los casos clínicos ha demostrado que persiste en la adolescencia y en la vida adulta impactando la funcionalidad del sujeto en las diferentes áreas de desempeño (Lischinsky, Torralva, Torrente \& Manes, 2008; Still \& Schoener, 1996).

Sobre la memoria operativa en los niños con trastorno por déficit de atención e hiperactividad (TDAH), Martinussen, Hayden, Hogg-Johnson y Tannock (2005) encontraron en su revisión que todos sus componentes se ven afectados en los niños con este trastorno. Al igual que los niños con autismo, el peor desempeño de los niños con TDAH se dio en el componente espacial de la memoria operativa específicamente en el almacenamiento espacial, en cuanto al componente verbal des criben déficits menores. Sin embargo; las investigaciones no son concluyentes, los hallazgos de otros 
estudios como el realizado por Pineda Alhucema y Puentes Rozo (2013), hipotetizan déficit en la memoria de trabajo asociada a dificultades en la teoría de la mente en las tareas de atribuciones de falsa creencia, dado que se requiere mantener la información en la mente un cierto lapso, para poder analizar y luego atribuir.

El pobre desempeño espacial en esta población se puede deber a que las tareas que evalúan esta parte del componente involucran el hemisferio derecho del cerebro, el cual se ha visto afectado en los niños con TDAH (Kwon, Reiss, \& Menon, 2002). Otra posible hipótesis es que las tareas visoespaciales son más demandantes que las verbales ya que requieren procesos menos automatizados y familiares y que por esto los niños con este trastorno presentan un desempeño peor. En conclusión, se ha comprobado la alteración de la memoria operativa en los niños con TDAH, quienes presentan disfunciones frontoestriales además de anormalidades en el sistema dopaminérgico, regiones importantes para el adecuado funcionamiento de la MO (Durton, 2003).

\subsection{Trastornos del Aprendizaje (TA)}

Aluden a cualquier forma de dificultad para el aprendizaje y en el sentido limitado, se refiere a una discapacidad específica para el aprendizaje (TEA). Este trastorno usualmente presenta alteraciones en las funciones ejecutivas del niño, específicamente en la memoria operativa.

Debido a que la memoria operativa tiene un rol importante en la comprensión del lenguaje, la inteligencia fluida, escritura, aritmética y la resolución de problemas es lógico considerar que los niños con trastornos específicos del aprendizaje (TEA) presentan alteración en este dominio cognitivo. Swanson, Ashbaker y Lee, 1996, Swanson y Beebe-Frankenberger (2004) y Swanson y Alloway (2011) han encontrado que los niños con inteligencia normal pero con dificultades en la lectura o en matemáticas se tienen que esforzar más en las tareas relacionadas con memoria operativa que sus pares que no presentan este trastorno. Siegel y Mazabel (1989) explican que los niños con dificultades de lectura presentan déficits en el procesamiento fonológico, impidiendo su habilidad para recuperar la información verbal de la memoria de corto plazo (Swanson, 2015). Sin embargo, estas dificultades en el bucle fonológico no parecen tener consecuencias en la inteligencia general del niño sino solo en aspectos relacionados con la lectura, indicando que tal vez la raíz de los problemas de aprendizaje en niños con dificultades de lectura se deba a déficits en este componente de la memoria operativa (Siegel \& Mazabel, 1989). La relación entre déficits en la agenda visoespacial y dificultades en la lectura y matemáticas todavía está inconcreta (Mammarella, Lucangeli, \& Cornoldi, 2010; Menghini, Finzi, Carlesimo, \& Vicari, 2011). Mientras que unos consideran que la agenda visoespacial se mantiene intacta en los niños con dificultades de lectura en comparación de sus pares (O'Shaughnessy \& Swanson, 1998), otros autores sugieren que bajo condiciones donde hay mucha demanda de procesamiento visoespacial, los niños con dificultades matemáticas obtienen un peor desempeño en comparación de sus pares de la misma edad. Con respecto al Ejecutivo Central (EC), el componente más comprometido en los niños con TEA es la atención controlada, específicamente presentan mayores déficits en el monitoreo y la actualización de la información, sin embargo, no presentan alteraciones en el planeamiento ni sostenimiento de la atención a través del tiempo (Swanson, 2015). En conclusión, el desempeño de los niños con TEA depende en gran parte del tipo de tarea y los materiales utilizados, especialmente se desempeñarán peor en las tareas que requieren el rápido reconocimiento de cadena de letras, números, palabras 
reales y pseudopalabras (Swanson, 2015) esto se debe a que estas actividades necesitan de la habilidad del niño para almacenar y/o acceder a la estructura del sonido del lenguaje.

\subsection{Trastornos de la Comunicación (TC)}

Se pueden dividir en dos: aquellos que afectan el discurso y aquellos que afectan el lenguaje. Aquellos niños que presentan alteraciones en el discurso presentan dificultades para la producción de sonidos, para el control de los sonidos que son producidos por la voz y para controlar el ritmo del discurso. Por otro lado, los niños con desórdenes en el lenguaje presentan dificultades para utilizar correctamente el lenguaje (fonología, morfología y sintaxis). Además presentan alteraciones en la semántica y pragmática del lenguaje (Montgomery, 2003).

Al igual que los niños con trastornos específicos del aprendizaje, los niños con trastornos de la comunicación presentan alteraciones en el bucle fonológico de la memoria operativa (Montgomery, 2003). Gathercole y Baddeley (1990) fueron los primeros en estudiar la relación entre el aspecto fonológico de la memoria operativa y las alteraciones del lenguaje cuando realizaron un estudio comparativo entre niños con TL y niños con un desarrollo normal donde demostraron que aquellos niños con este trastorno se les hacía más difícil repetir sílabas de pseudopalabras que al grupo control sugiriendo que los niños con TC presentaban una menor capacidad en bucle fonológico. Sin embargo, a pesar de presentar una menor capacidad en el BF, en investigaciones realizadas por Montgomery $(2000 a, 2000 b)$ se encontró que estos niños presentaban una habilidad de comprensión comparable a los niños de su edad con un desarrollo normal sugiriendo que los niños con TC sacrifican el recuerdo de palabras para mantener su buena comprensión. En general, los niños con TC tendrán más dificultades para aprender nuevas palabras y morfemas gramaticales, especialmente cuando estos se les presentan bajo condiciones estresantes de procesamiento; esto se debe a la dificultad de los niños de poder manejar tanto las capacidades de almacenamiento como de procesamiento de la MO (Montgomery, 2003). Como mencionamos anteriormente, los niños con este trastorno no presentan diferencias en su capacidad de comprensión, no obstante comparado con el grupo control producen una menor cantidad de palabras especialmente cuando están bajo un ritmo acelerado.

Se ha propuesto que un pobre bucle fonológico puede servir como marcador libre de cultura para el trastorno del lenguaje y que tareas como la repetición de pseudopalabras puede clasificar con precisión a los niños con TC de los niños con desarrollo normal (Campbell, Dollaghan, Needleman \& Janosky, 1997; Dollaghan \& Campbell, 1998; Ellis Weismer, Tomblin, Zhang, Buckwalter, Chyoweth \& Jones, 2000). Un aspecto importante a resaltar es que se ha encontrado que los niños con este trastorno parecen presentar una agenda visoespacial intacta (Gathercole \& Archibal, 2006; Schuchardt, Bockmann, Bornemann \& Maehler, 2013) y que esto se debe utilizar como una herramienta de apoyo para la capacitación de estrategias de memoria o apoyos visuales pertinentes a la hora de los programas de intervención.

\subsection{Discapacidad Cognitiva}

Se puede definir como aquella discapacidad que ocurre en el periodo de desarrollo, donde el niño nace con una capacidad intelectual por debajo del rango normal y que además presenta dificultades

Psicogente, 21 (39): 216-227. Julio-Diciembre, 2018. DOI http://doi.org/10.17081/psico.20.38.2543 
en el área adaptativa; puede ser en la comunicación, autocuidado, habilidades sociales, aprendizaje y autodirección (Criminal Justice Support Network, 2009).

Con respecto a la Discapacidad Cognitiva (DC), estudios han mostrado que los niños con DC tanto leve como moderada presentan problemas en la memoria operativa (Hulme \& Mckenzie, 1992; Jarrold \& Baddeley, 1997; Russel, Jarrold, \& Henry, 1996; Van der Molen, Van Luit, Jongmans, \& Van der Molen, 2007). Específicamente se ha visto que el componente más afectado es el bucle fonológico (Henry, 2001; Jarrold \& Baddeley, 1997; Rosenquist, Conners \& Roskos- Ewoldsen, 2003; Russel et al., 1996; Van der Molen et al., 2007). Como mencionamos anteriormente, tanto el BF como la agenda visoespacial están conformados por dos subcomponentes. El bucle fonológico presenta un almacén fonológico y un subsistema de recapitulación articulatorio; este último componente el que puede explicar algunos fenómenos como el "Efecto de similitud acústica", esto es, que el recuerdo inmediato de ciertos ítems es menor cuando las palabras tienen sonidos similares que cuando tienen sonidos distintos. La explicación a esto se debe a que el código base vinculado en el almacén es fonológico, los ítems similares tienen menos claves distintivas que los que no lo son haciendo que sean más fáciles olvidarlos. En cambio, las palabras con significado similar no tienen este efecto, lo que sugiere que el bucle fonológico no refleja un código semántico (Rosenquist et al., 2003). Otro fenómeno que se da en el BF es que el recuerdo inmediato se da mejor cuando las palabras son cortas (monosilábicas) que cuando son largas (multisilábicas), esto se llama el "Efecto de longitud de la palabra" y explica que los ítems que toman menos tiempo de articular son más ensayados y por ende, mejor recordados (Henry, 1991; Hulme \& Tordoff, 1989). Se ha encontrado que los niños con DC presentan una alteración en el "Efecto de la longitud de la palabra" en comparación con sus pares en edad mental sugiriendo una disminución o deficiencia en el subcomponente de recapitulación articulatorio (Hulme \& Mckenzie, 1992; Rosenquist et al., 2003). Sin embargo, es importante mencionar que en el estudio realizado por Rosenquist et al. (2003) los niños con DC recordaron igual cantidad de palabras tanto largas como cortas a diferencia del grupo control quienes recordaron más cantidad de palabras cortas. Una posible explicación es que debido a un reducido funcionamiento en el de recapitulación articulatoria, los niños con DC se apoyan más en la reintegración para el recuerdo de palabras. La reintegración, definida por Baddeley, Gathercole y Papagno (1998), es un proceso que utiliza la representación fonológica a largo plazo para rellenar o reconstruir una decadente memoria fonológica en el momento del recuerdo. Las palabras largas, teniendo una mayor información fonológica, se benefician de la reintegración más que las palabras cortas, haciéndolas más fáciles de recordar. Para los niños con DC, la combinación de una reintegración relativamente intacta y un reducido sistema de recapitulación articulatorio, puede haber ayudado a que recordaran igual cantidad de palabras largas como cortas en comparación con sus pares sin DC (Rosenquist et al., 2003).

Sobre la agenda visoespacial, es interesante ver que no se han encontrado alteraciones de este componente en los individuos con DC. Investigaciones como las de Rosenquist et al. (2003) han encontrado que tanto los niños con DC como niños de su misma edad mental requieren más tiempo y hacen más errores cuando hay más información visual que actualizar y que al igual, recuerdan más imágenes diferentes que similares.

\section{CONCLUSIONES}

Psicogente, 21 (39): 216-227. Julio-Diciembre, 2018. DOI http://doi.org/10.17081/psico.20.38.2543 
Después de la revisión realizada, se puede llegar a la conclusión que distintos componentes de la memoria operativa se ven afectados en los trastornos del neurodesarrollo. La importancia de esto radica en el hecho de que este proceso cognitivo tiene un papel relevante en el aprendizaje escolar (Sierra \& Ocampo, 2013); investigaciones como las de Alloway (2009) encontraron que las pruebas de $\mathrm{MO}$ predecían mejor el desempeño escolar que las pruebas de $\mathrm{Cl}$ y que la $\mathrm{MO}$ al inicio de la educación formal era un mejor predictor del éxito académico (Alloway, 2011). Al igual se ha encontrado que el bucle fonológico y la memoria operativa verbal tienen un papel influyente en la adquisición de la lectura, mientras que por otro lado la agenda visoespacial y el Ejecutivo Central son importantes para el rendimiento en tareas matemáticas, excepto cuando el contenido es numérico (Ocampo Gaviria \& Sierra Fitzgeald, 2014). Si bien se conoce que la MO se ve alterada en los TND, es necesario ampliar más sobre el funcionamiento de este proceso cognitivo en estos trastornos para poder comprender las diferencias de los niños en el aula de clasey poder brindarles mejores opciones terapéuticas que los ayuden a obtener un mejor rendimiento académico, además de desarrollar más sus fortalezas y mejorar sus debilidades.

Nota de Autores

Síndrome de Asperger en niños y adolescentes de Bucaramanga y Medellín Fase 1: Estudio de prevalencia y características cognitivas. 569 - 2012 Banco de Proyectos Elegibles de CTel.

\section{Referencias}

Alloway, T. P. (2009). Working memory, but not IQ, predicts subsequent learning in children with learning difficulties. Journal of Psychological Assessment, 25(2), 92-98. https://doi.org/10.1027/1015-5759.25.2.92

Alloway, T.P. (2011). A comparison of working memory profiles in children with ADHD and

DCD. Child Neuropsychology, 17(5), 483-494. https://doi.org/10.1080/09297049.2011.553590

American Psychiatric Association (2013). Diagnostic and statistical manual of mental disorders: DSM-5 (5thEd.). Arlington, Virginia: American Psychiatric Association.

Arteaga, G. \& Pimienta, H. (2006). Memoria operativa y circuitos corticales. Revista de la Facultad de Medicina Universidad Nacional Colombia, 54(4), 248- 268. Disponible en https://revistas.unal.edu.co/index.php/revfacmed/article/view/43940/64537

Baddeley, A.D. (2012). Working memory: theories, models, and controversies. Annual Review of Psychology, 63, 1-29. http://doi.org/10.1146/annurev-psych-120710-100422

Baddeley, A.D. \& Eysenck, M.W. (2010). Memoria. Madrid: Alianza Editorial.

Baddeley, A.D. \& Hitch, G. (1974). Working Memory. Psychology of Learning and Motivation, 8, 47-89. Doi: http://doi.org/10.1016/S0079-7421(08)60452-1

Baddeley, A.D., Gathercole, S.E. \& Papagno, C. (1998). The Phonological loop as a language learning device. Psychological Review, 105(1), 158-173. http://dx.doi.org/10.1037/0033-295X.105.1.158 
Campbell, T., Dollaghan, C., Needleman, H. \& Janosky, J. (1997). Reducing bias in language assessment:Processingdependent measures. Journal of Speech Language and Hearing Research, 40, 519-525. http://dx.doi.org/10.1044/jslhr.4003.519

Corbett, B., Constantine, L., Hendren, R., Rocke, D. \& Ozonoff, S. (2010). Examining executive funtioning in children with autism spectrum disorder, attention deficit hyperactivity disorder and typucal development. Psychiatry Res, 166(916), 210-222. Doi: http://doi.org/10.1016/j.psychres.2008.02.005.Examining

Criminal Justice Support Network (2009). Introduction to Intellectual Disability. Redfern, Australia: Intellectual Disability Rights Service.

Dollaghan, C. \& Campbell, T. (1998). Nonword repetition and child language impairment. Journal of Speech Language and Hearing Research, 41, 11136- 1146. http://dx.doi.org/10.1044/jslhr.4105.1136

Durton, S. (2003). A review of the biological bases of ADHD: what we have learned from imaging studies? Mental Retardation and Developmental Disabilities Research Reviews, 9(3), 184-195. http://dx.doi.org/10.1002/mrdd.10079

Ellis Weismer, S., Tomblin, B., Zhang, X., Buckwalter, P., Chyoweth, J. \& Jones, M. (2000). Nonword repetion performance in school-age children with and without language impairment. Journal of Speech, Language and Hearing Research, 43, 865-878. https://doi.org/10.1044/jslhr.4304.865

Etchepareborda, M.C. \& Abad-Mas, L. (2005). Memoria de trabajo en los procesos básicos del aprendizaje. Revista de Neurología, 40(1), 79-83. Recuperado de https://www.neurologia.com/articulo/2005078

Flores, J. \& Ostrosky-Shejet, F. (2012). Desarrollo neuropsicológico de lóbulos frontales y funciones ejecutivas. México: Manual Moderno.

Gathercole, S.E. \& Archibal, L.M. (2006). Visuoespatial Immediate Memory in Specific Language Impairment. Journal of Speech, Language and Hearing Research, 49, 265-277. https://doi.org/10.1044/10924388(2006/022)

Gathercole, S.E. \& Baddeley, A.D. (1990). Phonological memory deficits in language disordered children: Is there a causal connection? Journal of Memory and Language, 29, 336-360. Doi: https://doi.org/10.1016/0749596X(90)90004-J

Gathercole, S.E. \& Baddeley, A.D. (1993). Working Memory and Language. Cambridge: Lawrence Erlbaum Associates.

Goldstein, W., Carpenter, P. \& Minshew, N. (2005). Verbal and spatial working memory in autism. Journal of Autism and Developmental Disorders, 35(6), 747-56. https://doi.org/10.1007/s10803-005-0021-x

Henry, L.A. (1991). The effects of word lenght and phonemic similarity in young children's shortterm memory. Quarterly Journal of Experimental Psychology, 43(1), 35-52. Doi: http://dx.doi.org/10.1080/14640749108400998

Henry, L.A. (2001). How does the severity of a learning disability affect working memory performance? Memory, 9(46), 233-247. http://doi.org/10.1080/09658210042000085

Hulme, C. \& Mckenzie, S. (1992). Working Memory and Severe Learning Difficulties. Erlbaum, Hillsdale, NJ

Hulme, C. \& Tordoff, V. (1989). Working memory development: the effects of speech rate, word length, and acoustic similaritu on serial recall. Journal of Experimental Child Psychology, 47(1), 72-87. http://doi.org/10.1016/0022-0965(89)90063-5 
Xochitl Delgado Mckay, Maryoris Zapata Zabala.

Jarrold, C. \& Baddeley, A. D. (1997). Short-term memory for verbal and visouespatial information in Down's syndrome. Cognitive Neuropsychiatry, 2(2), 101-122. http://doi.org/10.1080/135468097396351

Julio, I. \& Gómez, C. (2010). Discapacidad en Colombia: Reto para la Inclusión en Capital Humano. Bogotá: Colombia Líder

Kercood, S., Grskovic, J. A., Banda, D. \& Begeske, J. (2014). Research in Autism Spectrum Disorders Working memory and autism: A review of literature. Research in Autism Spectrum Disorders, 8(10), 1316-1332. http://doi.org/10.1016/j.rasd.2014.06.011

Kwon, H., Reiss, A.L. \& Menon, V. (2002). Neural basis of protracted developmental changes in visuo-spatial working memory. Proceedings of the National Academy of Sciences of the United States of America, 99(20), 13336-41. http://doi.org/10.1073/pnas.162486399

Lischinsky, A., Torralva, T., Torrente, F. \& Manes, F. (2008). Trastorno por déficit de atención con hiperactividad en el adulto. In E. Labos, A. Slachevsky, P. Fuentes \& F. Manes (Eds.), Tratado de Neuropsicología Clínica (pp. 219-231). Buenos Aires: Akadia.

Mammarella, I. C., Lucangeli, D. \& Cornoldi, C. (2010). Spatial working memory and arithmetic déficits in children with nonverbal learning difficulties. Journal of Learning Disabilities, 43(5), 455-468. Doi: https://doi.org/10.1177/0022219409355482

Martinussen, R., Hayden, J., Hogg-Johnson, S. \& Tannock, R. (2005). A meta-analysis of working memory impairments in children with attentiondeficit/hyperactivity disorder. Journal of the American Academy of Child and Adolescent Psychiatry, 44(4), 377-84. http://doi.org/10.1097/01.chi.0000153228.72591.73

Menghini, D., Finzi, A., Carlesimo, G.A. \& Vicari, S.(2011). Working memory impairment in children with developmental dyslexia: Is it just a phonological deficit? Developmental Neuropsychology, 36(2),199-213. http://doi.org/10.1080/87565641.2010.549868

Montgomery, J.W. (2000a). Relation of working memory to off-line and real-time sentence processing in children with specific language impairment. Applied Psycholinguistics, 21(1), 117-148.

Montgomery, J.W. (2000b). Verbal working memory and sentence comprehension in children with specific language impairment. Journal of Speech Language and Hearing Research, 43(2), 293-308.

http://doi.org/10.1044/jslhr.4302.293

Montgomery, J.W. (2003). Working memory and comprehension in children with specific language

impairment: what we know so far. Journal of Communication Disorders, 36(3), 221-231. https://doi.org/10.1016/S0021-9924(03)00021-2

National Center for Learning Disabilities (2014). The State of Learning Disabilities: Facts, Trends and Emerging Issues (3rd ed.). New York: National Center for Learning Disabilities.

O' Shaughnessy, T. \& Swanson, H. L. (1998). Do imme- diate memory deficits in students with learning disabilities in reading reflect a developmental lag or deficit? A selective meta-analysis of the literature. Learning Disability Quarterly, 21(2), 123-148. https://doi.org/10.2307/1511341

Ocampo Gaviria, T. \& Sierra Fitzgeald, Ó. (2014). Análisis del funcionamiento de la memoria operativa en niños con trastornos en el aprendizaje 1. Acta Colombiana de Psicologia, 17(27), 81-90. http://doi.org/10.14718/ACP.2014.17.2.9 
Pineda Alhucema, W.F. \& Puentes Rozo, P. (2013). Estudio ex-post facto de la teoría de la mente en niños escolarizados diagnosticados con trastorno por déficit de atención-hiperactividad. Tesis Psicológica, 8(2), 144-161.

Pineda, M. (2014). Trastornos del espectro autista. Autism spectrum disorders. Anales de Pediatría, 81(1), 4-5. http://doi.org/10.1016/j.anpedi.2014.04.018

Rosenquist, C., Conners, F.A. \& Roskos-Ewoldsen, B. (2003). Phonological and Visuo-Spatial Working Memory in Individuals with Intellectual Disability. American Journal of Mental Retardation, 108(6), 403- 413. http://doi.org/10.1352/0895-8017(2003)108<403:PAVWMI>2.0.CO;2

Russel, J., Jarrold, C. \& Henry, L. (1996). Working memory in children with autism and with moderate learning difficulties. Journal of Child Psuchology and Psyquiatry, 37(6), 673-86. Recuperado de http://journals.sagepub.com/doi/abs/10.1177/0022219408315815

Saeed, T. (2011). A comparative Study of Working Memory in Children with Neurodevelopmental Disorders. (Tesis Doctoral). National University of Ireland Maynooth, Irlanda.

Schuchardt, K., Bockmann, A., Bornemann, G. \& Maehler, C. (2013). Working Memory Functioning in Children with Learning Disorders and Specific Language Impairment, 33(4),

298-312. http://doi.org/10.1097/01.TLD.0000437943.41140.36

Siegel, L.S. \& Mazabel, S. (1989). Basic cognitive processes and reading disabilities. In H.L. Swanson, K.R. Harris \& S. Graham (Eds.), Handbook of Learning Disabilities (pp. 158-181). New York: Guilford Press.

Sierra, Ó. \& Ocampo, T. (2013). El papel de la memoria operativa en las diferencias y trastornos del aprendizaje escolar. Revista Latinoamericana de Psicología, 45(1), 63-79. Recuperado de https://scholar.google.es/scholar?hl=es\&as_sdt=0\%2C5\&q=El+papel+de+la+memoria+operativa+en+las+dif erencias+y+trastornos+del+aprendizaje+escolar\&btnG=

Still, G. \& Schoener, E. (1996). Age-dependent decline of attention deficit hyperactivity disorder. American Journal of Psychiatry, 36(2), 159-165. http://doi.org/10.1017/S003329170500471X

Swanson, H.L. (2015). Intelligence, Working Memory and Learning Disabilities. In Cognition, Intelligence, and Achievement (pp. 173-196). Amsterdam: Elsevier. http://doi.org/10.1016/B978-0-12-410388-7.00010-5

Swanson, H.L. \& Alloway, T. (2011). Working memory, learning, and academic achievement. In K. Harris,T. Urban \& S. Graham (Eds.), APA Handbook of Educational Psychology. USA: APA.

Swanson, H.L. \& Beebe-Frankenberger, M. (2004). The relationship between working memory and mathematical problem solving in children at risk and not a risk for serious math difficulties. Journal of Child Experimental Psychology, 96, 471-491. http://dx.doi.org/doi:10.1037/0022-0663.96.3.471

Swanson, H.L., Ashbaker, M. \& Lee, C. (1996). Working-memory in learning disabled readers as a function of processing demands. Journal of Child Experimental Psychology, 61(3), 242-275.

http://dx.doi.org/doi:10.1006/jecp.1996.0016

Van der Molen, M.J., Van Luit, J.E.H., Jongmans, M.J., \& Van der Molen, M.W. (2007). Verbal working memory in children with mild intellectual disabilities. Journal of Intellectual Disability Research: JIDR, 51(2), 162-9. http://doi.org/10.1111/j.1365-2788.2006.00863.x 


\section{Xochitl Delgado Mckay, Maryoris Zapata Zabala.}

Vélez-Álvarez, C. \& Vidarte Claros, J. (2012). Trastorno por déficit de atención e hiperactividad (TDAH), una problemática a abordar en la política pública de primera infancia en Colombia. Revista de Salud Pública, 14(2), 113-128. https://doi.org/10.1590/S0124-00642012000800010 